\title{
U-Turning Ant Colony Algorithm for Solving Symmetric Traveling Salesman Problem
}

\author{
Saman M. Almufti ${ }^{1}$, Awaz A. Shaban ${ }^{2}$ \\ ${ }^{1}$ Department of Computer Science, Nawroz University, Duhok, Kurdistan Region - Iraq \\ ${ }^{2}$ Gara Institute,Duhok, Kurdistan Region - Iraq
}

\begin{abstract}
This paper provides a new Ant based algorithms called U-Turning Ant colony optimization (U-TACO) for solving a well-known NP-Hard problem, which is widely used in computer science field called Traveling Salesman Problem (TSP). Generally U-Turning Ant colony Optimization Algorithm makes a partial tour as an initial state for the basic conventional Ant Colony algorithm. This paper provides tables and charts for the results obtained by U-Turning Ant colony Optimization for various TSP problems from the TSPLIB95.

Keywords: Traveling Salesman Problem (TSP), Ant System (AS), Swarm Intelligence, U-Turning Ant Colony Optimization Algorithm (U-TACO), Symmetric Traveling Salesman Problem (STSP).
\end{abstract}

\section{Introduction}

In Computer science field, Swarm Intelligence (SI) algorithms are computational intelligence techniques that study the collective behavior in decentralized systems (Almufti, 2017). In the nature real-ants lives in colonies, Ants of a colony are cooperates the process of food searching. Ants are unsystematically travels searching for a food source, ones an ant reaches a food source it returns to the colony by using a chemical substance called pheromone trail that ant deposits it in the way to the food and can be smelled by other ant. UTurning Ant Colony Optimization algorithm is a new swarm intelligence algorithm based on the Ant colony algorithm in which ants randomly travel searching for the source of Food (Almufti, 2015). In this paper UTurning Ant Colony Optimization algorithm is used to solve Symmetric Traveling Salesman Problem in which a salesman want to visit all cities in the graph and return to the start city with minimum time and cost (Almufti, 2015; Asaad, R., Abdulnabi, N. 2018).

\section{Symmetric Traveling Salesman Problem (STSP)} TSP is one of widely used NP-hard problem in combinatorial optimization (Asaad, R., Abdulnabi, N. 2018). In a given graph a number of cities in which every city must be visited once and return to the starting city

Academic Journal of Nawroz University

(AJNU) Volume 7, No 4 (2018).

Regular research paper : Published 21 December 2018

Corresponding author's e-mail : saman.almofty@gmail.com

Copyright (02017 Saman M. Almufti1, Awaz A. Shaban2.

This is an open access article distributed under the Creative Commons Attribution License. for completing a tour such that the length of the tour is the shortest among all possible tours (Almufti, 2015; Andrej Kazakov, 2009). Symmetric TSP (STSP) is a type of TSP in which the distance between city A and B is equal to the distance between city $B$ and $A$.

In a graph $\mathrm{G}(\mathrm{C}, \mathrm{A})$ the distance $\mathrm{d}(\mathrm{Ci}, \mathrm{Cj})=\mathrm{d}(\mathrm{Cj}, \mathrm{Ci})$ and the number of tours in the Symmetric TSP (STSP) is (n1 )!/ 2 for $n$ cities. Consequently the optimal (minimum length) tour to the STSP can be obtained by finding the summation of the length between cities of a permutation list as shown in equation (1) (Almufti, 2015; Asaad, R., Abdulnabi, N. 2018).

$$
\text { Btour }=\left(\sum_{i=1}^{n-1} d_{\pi(P i) \pi(P i+1)}\right)+d_{\pi(P n) \pi(P 1)}
$$

Where $\mathrm{p}$ is a probability list of cities with minimum distance between cities (pi and pi+1) (Federico Greco 2008; Asaad, R., Abdulnabi, N. 2018).

\section{U-Turning Ant Colony Optimization (U-TACO)} U-Turning Ant Colony Optimization (U-TACO) is metaheuristic algorithm designed by Saman M. Almufti in his master thesis in (2015), it generally based on the same principles of common Ant System (AS) which was first represented in 1992 by Marco Dorigo in his PhD thesis as a nature-inspired metaheuristics for solving hard combinatorial optimization (CO) problems (Dorigo, 1992; Almufti, 2015; Almufti, 2017).

All algorithms that inspired the behavior of real Ant in searching for food source, basically depends on pheromone trail updating and the following of other ants to the pheromone smell: ants deposit pheromones in their way to the food source which takes attention of other swarm ants to the food source and the way that 
they should take to the food, gradually the way that have more pheromone is the shortest way to the food (Dorigo, 1992; Almufti, 2015) as shown in figure (1).

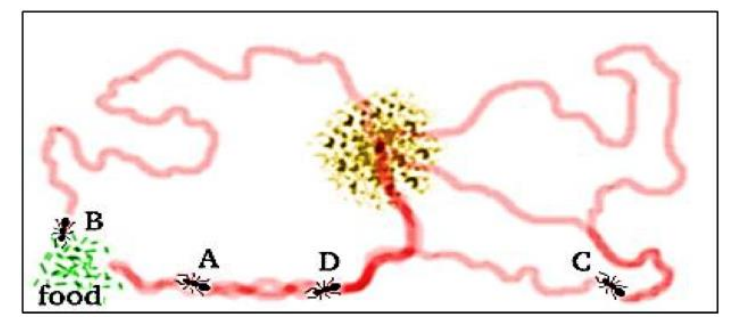

Figure 1: Behavior of real ants (Mutenet.sourceforge.net, 2015)

U-Turning Ant Colony Optimization (U-TACO) involve two phases:

Firstly: initializing pheromone matrix by UAnt to be used in second phase. UAnts makes a partial tour for the given graph and returns feedback in the same path as shown in figure (2), those partial tours leads to update the pheromone trail in the ways that seems to be useful in finding optimal tour by Ant (Almufti, 2015).

Secondly: finding the optimal or near-optimal tour for the problem by Ants, which make complete tours and goes back to the starting position as shown in figure (2).

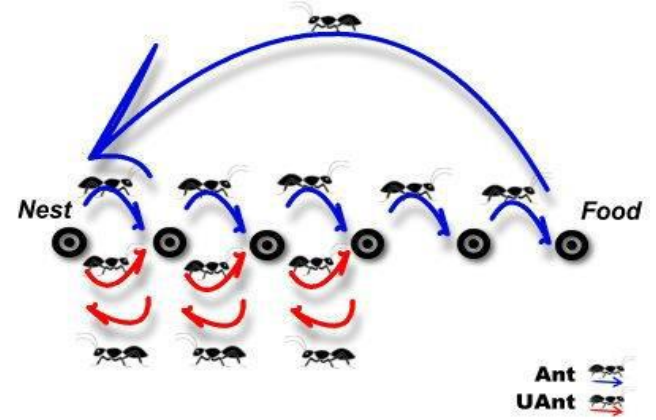

Figure 2: Ant and UAnt tour (Almufti, 2015) Uants are not responsible of finding an optimal solution for a given problem, where UAnt mission is only initializing the pheromone trail matrix, which will be use by ant to construct an optimal solution (Almufti, 2015).

\section{U-TACO and STSP}

The traveling salesman problem (TSP) is an important factor in testing and improving the Ant System (AS) algorithms and algorithms that based on the behavior of Ant (Almufti, 2015). The first appearance of Ant System by Marco Dorigo in 1992 was tested on the travelling salesman problem (Dorigo, 1992). As all Ant based algorithms U-Turning Ant Colony Optimization (UTACO) was first tested in TSP.

U-Turning Ant Colony Optimization (U-TACO) use Ant and UAnt in the process of finding the best tour for the Salesman, number of Ant is equal to the number of cities that the salesman want to visit them, where as the number of UAnt can take any value between 1 and number of cities. Both UAnts and Ants are following the same procedure in starting, updating pheromone trail and pheromone evaporation process, the only deferens occurs in the length and the way that it take back to the start city (Almufti, 2015).

The algorithm follows two phases; the first phase is making partial tours by UAnt which consist of the following steps.

1.Identify the length 1 that the UAnt should visit in the partial tour $(1<=1<=$ number of cities in graph).

2.Randomly allocate UAnts in cities.

Every UAnt chose the next city upon a probabilitybased function depend on pheromone trail and heuristic value, called random proportional rule (2), shows the probability of UAnt $(\mathrm{k})$ that locate at the city (i) to visit city (j) (Almufti, 2015).

$P_{i j}^{k}=\frac{\left[\tau_{i j}\right]^{\alpha} \cdot\left[\eta_{i j}\right]^{\beta}}{\sum_{1 \in \mathcal{N}_{i}^{k}}\left[\tau_{i j}\right]^{\alpha} \cdot\left[\eta_{i j}\right]^{\beta}}$

Where $\left(\eta \_i j\right)$ is a priori available heuristic value, (dij) is the distance between cities ( $i$ and $j),\left(\tau \_i j\right)$ is the pheromone trail matrix, $\alpha$ is a parameter to regulate the influence of $\tau_{-}$ij and $\beta$ is a parameter to regulate the influence of $\eta_{-} \mathrm{ij}$, and $\left(\mathcal{N}_{i}^{k}\right)$ is the set of the neighborhood that ant (k) haven't visit them yet (Almufti, 2015).

3.UAnt visit 1 cities.

4.Update pheromone trail matrix. Each UAnt after adding new city to its tour makes a local pheromone update as shown in (3) (Almufti, 2015).

$\tau_{i j}$ new $=\tau_{i j}$ old $+\Delta \tau_{i j}$

5.Gradually the pheromone evaporates in the path (Almufti, 2015).

$\mathrm{\tau}_{\mathrm{ij}}$ new $=(1-\psi) \cdot \mathrm{\tau}_{\mathrm{ij}}$ old

Whereas $(0<\psi<1)$ is a constant value used for reducing pheromone trail.

After all UAnts complete their partial tour and updating and evaporating the pheromone trail, the first phase complete and the algorithm goes in to the second phase: a Complete tour by Ant.

In the second phase the Ant follow the same steps (initialize, allocate, pheromone trail updates and evaporations) in the first phase but here the ant makes a complete tour and goes to the start city as shown in figure 3. 


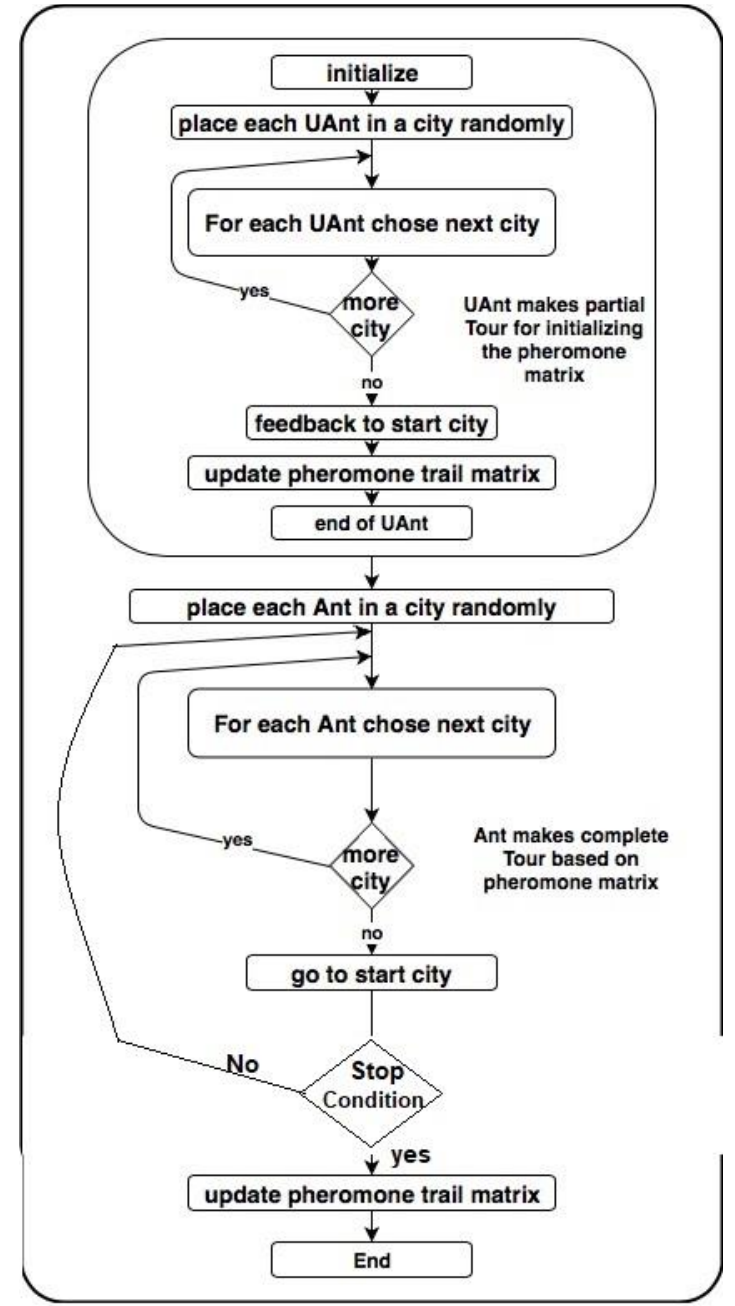

Figure 3: U-Turning Ant Colony flowchart

\section{Experimental Results:}

This section presents the performance of using UTurning Ant Colony Optimization (U-TACO) to solve STSP. The algorithms tested in some instances from the TSPLIB library (Karaboga D, Basturk B. 2007). The experiments have been performed on a PC with $2 \mathrm{GHz}$ processor and 8 GB RAM memory running MATLAB $\mathrm{R} 2015 \mathrm{~b}$ in windows 10. Each instance is run for 100 times.

UAnt can makes partial tour of different length between 1 and total number of city, here we tried three different value for tour length $1 / 4,1 / 2$, and $3 / 4$ of city numbers for different TSP problem. The results obtained in 50 iterations for UAnt to initialize pheromone trail matrix and 20 iterations for Ant to construct a complete tour. It was found from the results of (Lin105, Pr107, Ei151) STSP problem that U-TACO have the best results when UAnt make tour with length equal to $1 / 2$ of number of cities in the problem as shown in figure 4 , figure 5 , and figure 6 .

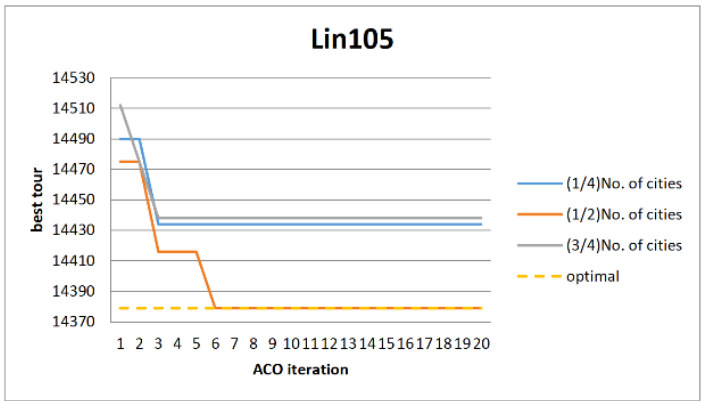

Figure 4: U-TACO for Lin105 over length $1 / 4,1 / 2$, and $3 / 4$ of city numbers

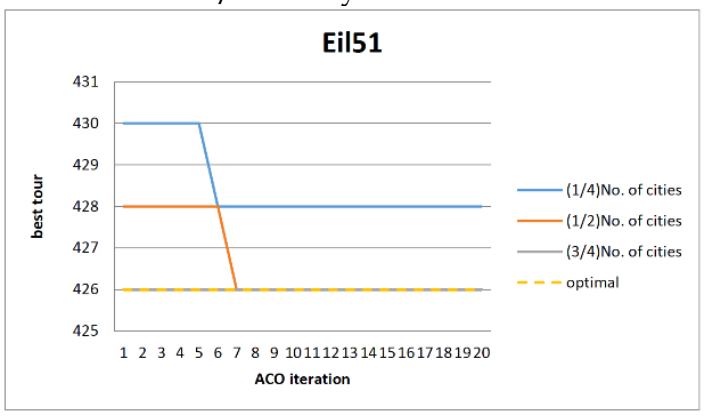

Figure 5: U-TACO for Eil51 over length 1/4, 1/2, and $3 / 4$ of city numbers

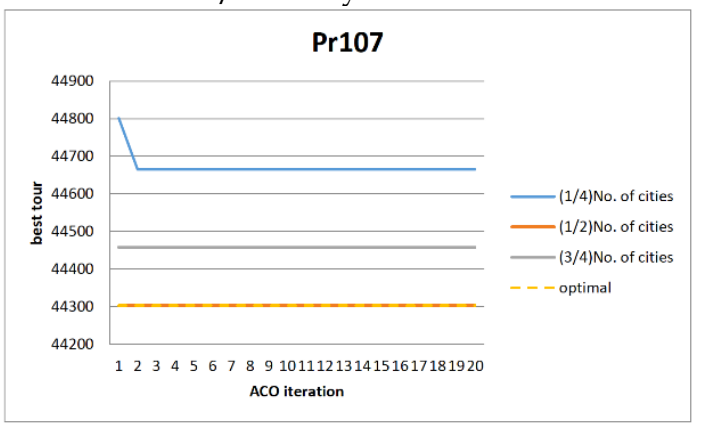

Figure 6: U-TACO for Pr107 over length 1/4, 1/2, and $3 / 4$ of city numbers

By considering figures (4, 5, and 6), in this paper simulate U-TACO when UAnt had make a partial tour of length $1 / 2$ * number of cities for the collection of STSP. 
Table 1: U-TACO results over 20 iteration

\begin{tabular}{|c|c|c|c|c|c|}
\hline $\begin{array}{c}\text { STSP } \\
\text { problem }\end{array}$ & $\begin{array}{l}\text { Optimal } \\
\text { Tour }\end{array}$ & U-TACO & Time & $\begin{array}{c}\text { \# of function } \\
\text { Evaluation }\end{array}$ & $\begin{array}{c}\text { Optimal } \\
\text { foundation }\end{array}$ \\
\hline att48 & 10628 & 10628 & 72.608541 & 210960 & $\begin{array}{c}\text { Iteration }=2 \\
\text { Time }=56.832731 \\
\text { Evaluation }=21096\end{array}$ \\
\hline att532 & 27686 & 28218 & $43566.032999 S$ & 2338140 & Not \\
\hline berlin52 & 7542 & 7542 & $92.900329 \mathrm{~s}$ & 228540 & $\begin{array}{c}\text { Iteration }=3 \\
\text { Time }=76.081437 \mathrm{~s} \\
\text { Evaluation }=34281\end{array}$ \\
\hline Lin105 & 14379 & 14379 & $467.574097 \mathrm{~s}$ & 461475 & $\begin{array}{c}\text { Iteration }=1 \\
\text { Time }=358.475811 \mathrm{~s} \\
\text { Evaluation }=23073\end{array}$ \\
\hline Ch150 & 6528 & 6547 & $1368.303153 \mathrm{~s}$ & 659250 & Not \\
\hline Eil51 & 426 & 427 & $75.529904 \mathrm{~s}$ & 224145 & Not \\
\hline Eil76 & 538 & 538 & $349.082318 \mathrm{~s}$ & 334020 & $\begin{array}{c}\text { Iteration }=7 \\
\text { Time }=313.814483 \mathrm{~s} \\
\text { Evaluation }=11690 \\
7\end{array}$ \\
\hline Eil101 & 629 & 629 & $439.032526 \mathrm{~s}$ & 443895 & $\begin{array}{c}\text { Iteration }=8 \\
\text { Time }=367.860212 \mathrm{~s} \\
\text { Evaluation }=17755 \\
8\end{array}$ \\
\hline $\mathrm{fl} 1417$ & 11861 & 12045 & 20596.700821s & 1832715 & Not \\
\hline kroA100 & 21282 & 21282 & $415.284487 \mathrm{~s}$ & 439500 & $\begin{array}{c}\text { Iteration }=6 \\
\text { Time }=344.404586 \mathrm{~s} \\
\text { Evaluation }=13185 \\
0\end{array}$ \\
\hline kroA150 & 26524 & 26618 & $1243.611930 \mathrm{~s}$ & 659250 & Not \\
\hline kroA200 & 29368 & 29472 & $2808.580653 \mathrm{~s}$ & 879000 & Not \\
\hline kroB100 & 22141 & 22200 & $439.673347 \mathrm{~s}$ & 439500 & Not \\
\hline kroB150 & 26130 & 26242 & $1333.134116 \mathrm{~s}$ & 659250 & Not \\
\hline kroC100 & 20749 & 20798 & $446.163972 \mathrm{~s}$ & 439500 & Not \\
\hline kroD100 & 21294 & 21455 & $434.844920 \mathrm{~s}$ & 439500 & Not \\
\hline Lin318 & 42029 & 42749 & $14079.427485 s$ & 1397610 & Not \\
\hline Pr76 & 108159 & 108487 & $202.229656 \mathrm{~s}$ & 334020 & Not \\
\hline Pr107 & 44303 & 44303 & $524.576606 \mathrm{~s}$ & 470265 & $\begin{array}{c}\text { Iteration }=1 \\
\text { Time }=392.417070 \mathrm{~s} \\
\text { Evaluation }=23513\end{array}$ \\
\hline Pr124 & 59030 & 59030 & $680.470220 \mathrm{~s}$ & 544980 & $\begin{array}{c}\text { Iteration }=5 \\
\text { Time }=545.294855 \mathrm{~s} \\
\text { Evaluation }=13624 \\
5\end{array}$ \\
\hline Pr136 & 96772 & 100702 & $884.805854 \mathrm{~s}$ & 597720 & Not \\
\hline Pr144 & 58537 & 58669 & $1155.302216 \mathrm{~s}$ & 632880 & Not \\
\hline Pr152 & 73682 & 73867 & $1550.821031 \mathrm{~s}$ & 668040 & Not \\
\hline Rat99 & 1211 & 1213 & $981.875703 \mathrm{~s}$ & 435105 & Not \\
\hline Rd100 & 7910 & 7935 & $479.834143 \mathrm{~s}$ & 439500 & Not \\
\hline St70 & 675 & 679 & $212.419589 \mathrm{~s}$ & 307650 & Not \\
\hline Tsp225 & 3916 & 3928 & $3400.762717 \mathrm{~s}$ & 88500 & Not \\
\hline
\end{tabular}


Table (1) presents the simulation results (tour length, time elapsed and number of function evaluation) for solving 27 STSP problems from the TSPLIB95 library by using U-TACO Algorithm. All problems were solved with 20 U-TACO iterations and the parameter that determine the influence of pheromone trail $a=1$ and heuristic information $\beta=2$, for each TSP problem number of ants equal to number of cities over partial tour of length $1 / 2$ * number of cities for the collection of STSP, most of the simulation results were nearoptimal and the optimal value for 6 STSP (att48, berlin52, Lin105, Eil76, Eil101, kroA100, Pr107, and Pr124) problems are founded.

\section{Conclusion}

U-Turning Ant Colony Optimization U-TACO) is used to solve symmetric TSP problems over 50 iterations for UAnts and 20 iterations for Ants, the results were very good (optimal / near optimal) with a small difference between the optimal tour. But generally using U-TACO takes a long time in solving the problem and finding the number of function evaluations.

\section{References}

Almufti, S. (2015). U-Turning Ant Colony Algorithm powered by Great Deluge Algorithm for the solution of TSP Problem. [online]
Hdl.handle.net. Available at: http://hdl.handle.net/11129/1734 [Accessed 5 Aug. 2018].

Almufti, S. (2017). Using Swarm Intelligence for solving NPHard Problems. Academic Journal of Nawroz University, 6(3), pp. 4650. doi:10.25007/ajnu.v6n3a78

Andrej Kazakov, (2009), Travelling Salesman Problem: Local Search and Divide and Conquer working together

Asaad, R., Abdulnabi, N. (2018). Using Local Searches Algorithms with Ant Colony Optimization for the Solution of TSP Problems. Academic Journal Of Nawroz University, 7(3), 1-6. doi:10.25007/ajnu.v7n3a193

Dorigo, M. (1992). Optimization, Learning and Natural Algorithms, PhD thesis, Politecnico di Milano, Italy.

Federico Greco, (2008), Travelling Salesman Problem . Mute-net.sourceforge.net, (2015). MUTE: Simple, Anonymous File Sharing. [online] Available at: http://mute-

net.sourceforge.net/howAnts.shtml [Accessed 20 July. 2018] . 